2. - The orthogonal development of non-linear functionals in series of FourierHermite functionals, Ann. of Math. vol. 48 (1947) pp. 385-392. 642.

3. - Non-linear integral equations, Ann. of Math. vol. 51 (1950) pp. 629-

4. S. Kaczmarz and H. Steinhaus, Theorie der Orthogonalreihen, Warsaw-Lwow, 1935.

5. R. E. A. C. Paley, N. Wiener, and A. Zygmund, Notes on random functions, Math. Zeit. vol. 37 (1933) pp. 647-668.

UNIVERSITY OF MINNESOTA AND

MassachusetTs Institute of TeChNology

\title{
THE MULTIPLIER RULE FOR ORDINARY DIFFERENTIAL EQUATIONS
}

\section{PHILIP COOPERMAN}

One of the unsolved problems of the calculus of variations is to find a proof of the multiplier rule for the case of partial differential equations as side conditions. The essential difficulty lies in the fact that the theory of partial differential equations is not sufficiently developed to allow the use of the same proecedure which worked in the case of ordinary differential equations. Hence, it would be desirable to have a proof of the multiplier rule which made no appeal to the theory of differential equations, even in the case of ordinary differential equations. A proof of this type is the object of this paper.

Let us consider the problem of making stationary the functional $J[y]$ defined by

$$
J[y]=\int_{x_{0}}^{x_{1}} F\left(x, y_{1} \cdots y_{n}, y_{1}^{\prime} \cdots y_{n}^{\prime}\right) d x
$$

under boundary conditions which need not be specified here and under side conditions

$$
G_{j}\left(x, y_{1} \cdots y_{n}, y_{1}^{\prime} \cdots y_{n}^{\prime}\right)=0, \quad j=1, \cdots, p<n .
$$

The usual proof of the multiplier rule starts from this problem and shows that the problem of making stationary the functional $K[y, \lambda]$,

$$
K[y, \lambda]=\int_{x_{0}}^{x_{1}}\left\{F+\lambda_{j} G_{j}\right\} d x
$$

Received by the editors May 16, 1951. 
under the same boundary conditions has the same solution, $u_{1} \cdots$ $u_{n}$, and that $K[y, \lambda]$ and $J[y]$ have the same stationary value.

Instead of following this approach, let us start with the second problem and try to show that it is equivalent to the first. Equating the first variation of $K[y, \lambda]$ with respect to the $y_{i}$ to zero gives

$$
a_{i j} \lambda_{j}^{\prime}=b_{i j} \lambda_{j}+c_{i}, \quad i=1, \cdots, n ; j=1, \cdots, p,
$$

where

$$
a_{i j}=\frac{\partial G_{j}}{\partial y_{i}^{\prime}}, \quad b_{i j}=\frac{\partial G_{j}}{\partial y_{i}}-\frac{d}{d x} \frac{\partial G_{j}}{\partial y_{i}^{\prime}}, \quad c_{i}=\frac{\partial F}{\partial y_{i}}-\frac{d}{d x} \frac{\partial F}{\partial y_{i}^{\prime}}
$$

and the arguments of the functions are $x, u_{1} \cdots u_{n}, u_{1}^{\prime} \cdots u_{n}^{\prime}$. Performing the same operation on $K[y, \lambda]$ with respect to the $\lambda_{j}$ gives

$$
G_{j}\left(x, u_{1} \cdots u_{n}, u_{1}^{\prime} \cdots u_{n}^{\prime}\right)=0 .
$$

The equations (4) are $n$ in number, but involve $p$ functions $\lambda_{j}$. Hence, if the problem involving $K[y, \lambda]$ is to be meaningful, certain compatibility conditions must be satisfied. Let $A$ be the determinant of the quantities $a_{h j}(h, j=1, \cdots, p)$ and let us make the inessential assumption $A \neq 0$. Then, the first $p$ of the equations (4) can be put into diagonal form, namely

$$
\lambda_{j}^{\prime}=B_{h j} \lambda_{h}+C_{j}
$$

where the $B_{h j}$ involve the $a_{h j}$ and $b_{h j}$, and the $C_{j}$, the $a_{h j}$ and $c_{j}$. Substituting (7) in the remaining $n-p$ equations of (4), we arrive at

$$
\left(a_{k j} B_{h j}-b_{k h}\right) \lambda_{h}=c_{k}-a_{k j} C_{j}, \quad k=p+1, \cdots, n .
$$

Let the rank of the equations (8) be $r_{1}$. It is clear that $r_{1} \leqq \min (p, n-p)$. Then $n-p-r_{1}$ linear relations not involving the $\lambda_{h}$ can be found among the expressions on the right-hand side of (8). These relations are necessary conditions on the functions $u_{1} \cdots u_{n}$. Furthermore, $r_{1}$ of the functions $\lambda_{h}$ can be expressed as linear combinations of the remaining $p-r_{1} \lambda_{h}$. By means of these last relations, the equations ( 7 ) can be reduced to $p-r_{1}$ differential equations in the same number of variables and $r_{1}$ finite linear equations in $p-r_{1}$ variables.

We now have to deal with equations having the same form as (7) and (8). Hence the process indicated in the previous paragraph can be applied again to give $r_{1}-r_{2}$ necessary conditions on the $u_{1} \cdots u_{n}$, $r_{2}$ of the $\lambda_{h}$ can be expressed in terms of the remaining $p-r_{1}-r_{2} \lambda_{h}$, and we shall be left with $p-r_{1}-r_{2}$ equations of the type of $(7)$, and $r_{2}$ 
equations of the type of (8). For each step, this information can be put in the form of a line in a table.

\begin{tabular}{c|c|c|c|c|c} 
Step & $\begin{array}{c}\text { No. of } \\
\text { Diff. } \\
\text { Equations }\end{array}$ & $\begin{array}{c}\text { No. of } \\
\text { Finite } \\
\text { Equations }\end{array}$ & $\begin{array}{c}\text { Rank of } \\
\text { Finite } \\
\text { Equations }\end{array}$ & $\begin{array}{c}\text { No. of } \\
\text { Conditions } \\
\text { on } u_{1} \cdots u_{n}\end{array}$ & $\begin{array}{c}\text { No. of } \\
\text { Conditions } \\
\text { on } \lambda_{1} \cdots \lambda_{p}\end{array}$ \\
\hline 1 & $p$ & $n-p$ & $r_{1}$ & $n-p-r_{1}$ & $r_{1}$ \\
2 & $p-r_{1}$ & $r_{1}$ & $r_{2}$ & $r_{1}-r_{2}$ & $r_{2}$ \\
3 & $p-r_{1}-r_{2}$ & $r_{2}$ & $r_{3}$ & $r_{2}-r_{3}$ & $r_{3}$ \\
. & & & & & \\
$\dot{m}$ & $p-r_{1}-\cdots-r_{m-1}$ & $r_{m-1}$ & $r_{m}$ & $\frac{r_{m-1}-r_{m}}{n-p-r_{m}}$ & $r_{m}+\cdots+r_{m}$
\end{tabular}

This process can end in one of two ways, namely, either by the number of differential equations, or by the number of finite equations reducing to zero. If, at the $(m+1)$ st step, the first alternative occurs, then a glance at the table shows that

$$
p=r_{1}+\cdots+r_{m}
$$

But then the table shows that we shall have exactly $p$ independent relations among the $\lambda_{j}$, thus determining them uniquely as functions of $u_{1} \cdots u_{n}$. By substituting those $\lambda_{j}$ determined at the $m$ th step in the differential equations which occurred at that step, we shall obtain $p-r_{1}-\cdots-r_{m-1}=r_{m}$ differential equations in the $u_{1} \cdots u_{n}$. These, together with those already obtained, are $n-p$ in number. Combining the $n-p$ conditions and the equations (6), we shall have a system of $n$ differential equations for the functions $u_{1} \cdots u_{n}$.

In the event that the number of finite equations vanish at the $(m+1)$ st step, it would follow that $r_{m}=0$. The table shows that we would have exactly $n-p$ conditions on the $u_{1} \cdots u_{n}$ in this case. On the other hand, we would have $r_{1}+\cdots+r_{m-1}$ finite equations in the $\lambda_{j}$ and $p-r_{1}-\cdots-r_{m-1}$ differential equations. Hence, the $\lambda_{j}$ would be determined by the $u_{1} \cdots u_{n}$ only up to $p-r_{1}-\cdots-r_{m-1}$ arbitrary constants. These results, in contrast to the preceding case, do, however, depend on the theory of differential equations.

In either case, it is clear that if functions $u_{1} \cdots u_{n}$ exist which satisfy the $n-p$ compatibility conditions, then also functions $\lambda_{1} \ldots$ $\lambda_{p}$ exist. If in addition, the $u_{1} \cdots u_{n}$ satisfy the $p$ equations (6), and the boundary conditions, the functional $K[y, \lambda]$ actually attains its stationary value. Consider the following remark due to $\mathrm{R}$. Courant 
(see reference). If a functional $J[u, v, \cdots]$ is stationary under certain side conditions for a given set of functions, and if this set of functions satisfies one or more relations, then $J$ remains stationary for the same set of functions if one or more of these relations are added to the side conditions.

Now, by means of this remark, we can add the equations (6) to the side conditions of the problem. The functional $K[y, \lambda]$ turns into $J[y]$ and we are returned to the original problem. It is clear that the two problems have the same solution $u_{1} \cdots, u_{n}$, and the same stationary value. Since it has already been shown that the appropriate $\lambda_{1} \cdots \lambda_{p}$ exist, both problems are meaningful. Hence, the multiplier rule is proved.

The author has already applied these ideas to the case of partial differential equations and has obtained some results. These will be published in a later paper.

\section{REFERENCE}

R. Courant and D. Hilbert, Methoden der mathematischen Physik, vol. 1, New York, Interscience, p. 201.

Research Corporation, Bound Brook, N. J. 